\title{
Can a Species Keep Pace with a Shifting Climate?
}

\author{
H. Berestycki ${ }^{\mathrm{a}, *}$, O. Diekmann ${ }^{\mathrm{b}}$, C.J. Nagelkerke ${ }^{\mathrm{c}}$, P.A. Zegeling ${ }^{\mathrm{b}}$ \\ ${ }^{a}$ École des hautes études en sciences sociales, CAMS, 54 Boulevard Raspail, 75006 Paris, \\ France \\ ${ }^{b}$ Department of Mathematics, Utrecht University, P.O. Box 80010, 3508 TA Utrecht, \\ The Netherlands \\ ${ }^{c}$ Institute for Biodiversity and Ecosystem Dynamics (IBED), University of Amsterdam, \\ Kruislaan 320, 1098 SM Amsterdam, The Netherlands
}

Received: 15 July 2007 / Accepted: 23 October 2008 / Published online: 9 December 2008 (C) The Author(s) 2008. This article is published with open access at Springerlink.com

\begin{abstract}
Consider a patch of favorable habitat surrounded by unfavorable habitat and assume that due to a shifting climate, the patch moves with a fixed speed in a onedimensional universe. Let the patch be inhabited by a population of individuals that reproduce, disperse, and die. Will the population persist? How does the answer depend on the length of the patch, the speed of movement of the patch, the net population growth rate under constant conditions, and the mobility of the individuals? We will answer these questions in the context of a simple dynamic profile model that incorporates climate shift, population dynamics, and migration. The model takes the form of a growth-diffusion equation. We first consider a special case and derive an explicit condition by glueing phase portraits. Then we establish a strict qualitative dichotomy for a large class of models by way of rigorous PDE methods, in particular the maximum principle. The results show that mobility can both reduce and enhance the ability to track climate change that a narrow range can severely reduce this ability and that population range and total population size can both increase and decrease under a moving climate. It is also shown that range shift may be easier to detect at the expanding front, simply because it is considerably steeper than the retreating back.
\end{abstract}

Keywords Climate change $\cdot$ Reaction-diffusion equation $\cdot$ Traveling wave $\cdot$ Moving favorable patch $\cdot$ Co-moving population profile $\cdot$ Persistence $\cdot$ Extinction $\cdot$ Principal eigenvalue

\section{Introduction}

The area occupied by a species is to a large extent determined by the climatic circumstances with temperature playing a major role. The global warming phenomenon, there-

\footnotetext{
*Corresponding author.

E-mail address: hb@ehess.fr (H. Berestycki).
} 
fore, has a great impact on survival and location of such species. See Walther et al. (2002) for a review of ecological responses to recent climate change.

We idealize the world by putting the North Pole at $+\infty$ and the equator at $-\infty$. This ignores the finiteness of the Earth, but it offers a good framework for a theoretical analysis. Warming and its effect can be seen as a shift in the profile of local climatic suitability, which the population density profile of a species tries to track. If a species keeps pace, its area expands as much in the north as it loses in the south. However, if it lags behind too much, it will go extinct.

Which of these two scenarios applies? How does the answer depend on the mobility of the species, on the extensiveness of the area, the local population dynamics, and on the speed of climate shift and the way climate actually acts on a species? If the species survives, what happens to the size and form of its population profile?

The recent research of one of us (Nagelkerke, 2004) tackles these issues in the context of a relatively realistic metapopulation model, using simulations as the main tool. The aim of the present paper is to address the same issues for a continuous population using an analytical approach. We study a simplified model, taking the form of a reactiondiffusion equation. Within this framework, our findings confirm the ones that had been observed in simulations. Here, we establish these results for a large class of equations, with rigorous mathematical proofs, thus proving their robustness and shedding light on the mathematical properties behind them.

From an ecological point of view, our main results are the following:

- An explicit formula (23) and in different forms in (24) and (25), for the critical size of the favorable patch for persistence, as a function of the Malthusian parameters, the diffusion constant and the climate speed. The formula pertains to the juxtaposition of two types of homogeneous habitat, the favorable patch being a bounded interval outside of which the environment is unfavorable.

- Revelation of a striking asymmetry in the comoving population profile: the north front is much steeper than the south tail and the population maximum occurs near to the northern border of the population profile.

- The observation that if the climate does not move too fast, the size of the total population as well as the range of the population may actually grow, relative to the situation in a static climate. But when the climate speed is further increased, an abrupt collapse may follow (see Figs. 7, 8 and 9).

The model we study here takes the form of the following reaction-diffusion equation:

$$
\frac{\partial u}{\partial t}=D \frac{\partial^{2} u}{\partial x^{2}}+f(u, x-c t) .
$$

Here, $-\infty<x<+\infty$ and $c$ is a given positive number. Here, $u$ is the population density of the species of interest and we have assumed that dispersal is adequately described by diffusion with constant $D$. The function $f$ describes the net effect of reproduction and mortality and how this depends on population density and on the local climatic conditions. Hence, it expresses the suitability profile. Note that $D$ is assumed to be independent of climate. The situation before the climate shift sets in corresponds to $c=0$. We assume that

$$
f(u, x)=u g(u, x),
$$


where the per capita growth rate $g$ is negative for large values of $x$, both negative and positive. More precisely, we shall incorporate only negative density dependence in the model (i.e., we do not incorporate an Allee effect, as discussed in Shi and Shivaji, 2006 for instance) and so the suitable area is

$$
\{x: g(0, x)>0\}
$$

which we assume to be an interval of length $L$.

The key questions concerning (1) are: does a positive solution of the form

$$
u(t, x)=w(x-c t)
$$

exist and is it a stable solution? What is the form of the solution? If no such solution exists, does it follow that $u$ converges to zero for $t \rightarrow \infty$ ? How do the answers depend on $c, D, L$, and other parameters characterizing $f$ ?

Such questions are a bit reminiscent of the "critical patch length" problem (cf. Okubo and Levin, 2001 and Ludwig et al., 1979), the "traveling wave invasion" problem (cf. Kolmogorov et al., 1937; Fisher, 1937; Aronson and Weinberger, 1978; Berestycki and Hamel, 2009; Thieme and Zhao, 2003; Rass and Radcliffe, 2003) and the "heterogeneous environment" problem (cf. Berestycki et al., 2005a, 2005b; Roques and Stoica, 2007; Shigesada and Kawasaki, 1997; Shigesada et al., 1986; Weinberger, 2002). Yet, the mix of ingredients (in particular, the fact that $c$ is prescribed, and hence amounts to an external forcing) makes it different from each of these and apart from Pease et al. (1989), where a quantitative genetics approach is adopted, we could not find any references. After most of the work described here was finished, however, we came across the preprint version of Potapov and Lewis (2004), which addresses exactly the same question, but with emphasis on the effect of a moving climate on the outcome of competitive interaction between two species. In fact, the special case that we treat in Sections 2 and 3 is also treated by Potapov and Lewis. Yet, we decided to include our analysis of this case in this paper as (1) the method is more geometrical (essentially phase plane analysis), (2) we deliver an analytical solution for the population profile and (3) we present additional results, leading to further biological insights. Other related work can be found in Dahmen et al. (2000), Deasi and Nelson (2005), and Pachepsky et al. (2005). It is known that diffusion enhances invasion speed but is counter productive for population growth on a finite stationary patch. Consequently, for a moving patch, there is a conflict between gain due to colonization of newly favorable habitat and loss due to migration into unfavorable habitat. A key result, formula (23) below, provides a quantitative algorithm for deciding which of these two effects is the stronger one.

We employ two different methods. If we assume that $g$, as a function of $x$, is piecewise constant we can glue phase portraits corresponding to the second order traveling wave ODE:

$$
D w_{\xi \xi}+c w_{\xi}+w g(w, \xi)=0, \quad \xi=x-c t .
$$

Making use of the linearization at $w=0$ we thus derive rather explicit answers to the key questions.

A more qualitative analogue of these answers for quite general $g$ is obtained by a PDE approach. The information provided by the linearization at zero is again crucial. Using 
various methods, notably the comparison principle, we derive, in Section 4, a dichotomy from this information:

- Either no positive traveling wave exists and zero is the global attractor,

- Or such a wave does exist and it attracts all orbits starting from nonnegative $(\not \equiv 0)$ initial data.

The biological insights derived from our analysis are explained in detail in Sections 2 and 3 while taking for granted that the results of Section 4 demonstrate the correctness and the robustness of the conclusions. More ecological consequences are discussed in Section 5. Readers who are looking for theorems and proofs will find the rigorous theory for a general class of equations presented in Section 4.

\section{Glueing phase portraits}

Throughout this section, we assume that for given positive parameters $\tilde{r}, r, K$, and $L$,

$$
g(u, x)= \begin{cases}-\tilde{r} & x<0 \text { and } x>L, \\ r\left(1-\frac{u}{K}\right) & 0 \leq x \leq L,\end{cases}
$$

while requiring that solutions are $C^{1}$ (indeed, to guarantee that diffusion conserves mass, the flux $D \frac{\partial u}{\partial x}$ should be continuous). In this model, the underlying assumption is that spatial heterogeneity is fully described by two abrupt changes taking places at the positions $x=0$ and $x=L$. By scaling $u, t$, and $x$, we can reduce the number of parameters from six to three. We choose to do this in such a way that the new values of $K, \tilde{r}$, and $D$ are all one. To facilitate the interpretation of our final results, we list how the new $r, c$, and $L$ relate to the six original parameters:

$$
\begin{aligned}
& r_{\text {new }}=\frac{r_{\text {old }}}{\tilde{r}}, \\
& L_{\text {new }}=\sqrt{\frac{\tilde{r}}{D}} L_{\text {old }}, \\
& c_{\text {new }}=\frac{1}{\sqrt{\tilde{r} D}} c_{\text {old }}, \\
& w_{\text {new }}=\frac{w_{\text {old }}}{K} .
\end{aligned}
$$

Already at this stage we can conclude that $K$ only sets the scale for $u$, but that it irrelevant for the answers to our questions.

In the outer regions $\xi<0$ and $\xi>L$, the function $w$ that we seek to construct should satisfy the linear equation

$$
w_{\xi \xi}+c w_{\xi}-w=0 .
$$






Fig. 1 The unstable and the stable subspace (restricted to $w>0$ ) for the linear system (10).

Define

$$
\mu_{ \pm}=-\frac{c}{2} \pm \sqrt{1+\left(\frac{c}{2}\right)^{2}}
$$

then any solution to (8) is a linear combination of $\exp \left(\mu_{+} \xi\right)$ and $\exp \left(\mu_{-} \xi\right)$. Since $\mu_{+}>0$ and $\mu_{-}<0$ and we want $w$ to be bounded, the solution for $\xi<0$ should be a multiple of $\exp \left(\mu_{+} \xi\right)$ while the solution for $\xi>L$ should be a multiple of $\exp \left(\mu_{-} \xi\right)$.

If we rewrite (8) as the first order system:

$$
\begin{aligned}
& w_{\xi}=v, \\
& v_{\xi}=w-c v,
\end{aligned}
$$

and think in terms of orbits in $(w, v)$-space, the solution for $\xi<0$ corresponds to motion away from the origin along the half-line $v=\mu_{+} w, w>0$, while the solution for $\xi>L$ corresponds to motion toward the origin along the half-line $v=\mu_{-} w, w>0$ (see Fig. 1). The analogue of (10) for $0 \leq \xi \leq L$ is

$$
\begin{aligned}
& w_{\xi}=v, \\
& v_{\xi}=-r w(1-w)-c v .
\end{aligned}
$$

This system has equilibria $(w, v)=(0,0)$ and $(w, v)=(1,0)$. (Incidentally, orbits connecting these two equilibria yield the classical KPP-Fisher traveling waves Fisher, 1937; Kolmogorov et al., 1937. These exist if and only if $c \geq 2 \sqrt{r}$. The lowest possible wave speed, $2 \sqrt{r}$, is the invasion speed Aronson and Weinberger, 1978; Berestycki and Hamel, 2009.) 


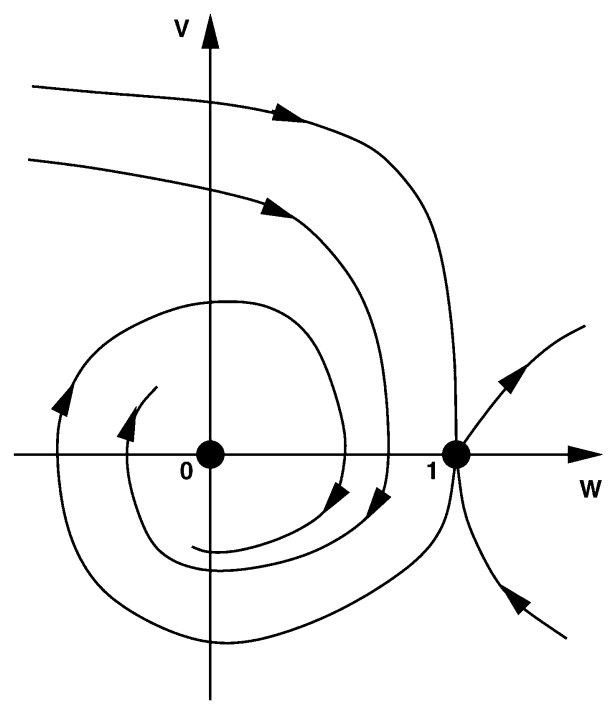

Fig. 2 Phase portrait of (11) for $\left(\frac{c}{2}\right)^{2}<r$.

The linearization at $(1,0)$ has eigenvalues $-\frac{c}{2} \pm \sqrt{r+\left(\frac{c}{2}\right)^{2}}$. So one is positive and the other negative or, in other words, $(1,0)$ is a saddle point. The linearization at $(0,0)$ has eigenvalues:

$$
\sigma_{ \pm}=-\frac{c}{2} \pm \sqrt{-r+\left(\frac{c}{2}\right)^{2}}
$$

Provided $-r+\left(\frac{c}{2}\right)^{2}<0$ these form a complex conjugate pair and then since $c>0$, $(0,0)$ is a stable spiral point (see Fig. 2).

If on the other hand, $-r+\left(\frac{c}{2}\right)^{2}>0$, then $(0,0)$ is a stable node. Since $\mu_{-}<\sigma_{ \pm}$, orbits of (10) that approach the origin from the positive half plane $w>0$ do so "above" the line $v=\mu_{-} w$. It is known (see Hadeler and Rothe, 1975; Aronson and Weinberger, 1978; Volpert et al., 1994, or Diekmann and Temme, 1982) that the unstable manifold of $(1,0)$ that lies in the region $w \leq 1$ does, in fact, approach the origin in this manner, and that it lies entirely above the line $v=\mu_{-} w$ (in fact above $v=\sigma_{-} w$ ).

Our task is to make a connection between the line $v=\mu_{+} w$ and the line $v=\mu_{-} w$ by way of a piece of orbit of (11) that is completed in a $\xi$-interval of exactly length $L$. The preceding paragraph established that this is impossible for $\left(\frac{c}{2}\right)^{2}>r$, since then the connecting orbit between $(1,0)$ and $(0,0)$ forms an obstruction. (Note that this implies the nonsurprising fact that a species can never track a climate that moves faster than the invasion speed of that species into the favorable habitat.) So, we focus our attention on the situation obtained by combining Figs. 1 and 2 (see Fig. 3).

In view of the results of Schaaf on two-point boundary value problems (Schaaf, 1990), it is to be expected that the length of the $\xi$-interval of an orbit piece connecting the two lines increases with increasing distance (along either line) from the origin (with limit $+\infty$ if we approach the connection via pieces of the stable - and unstable manifold of $(1,0)$ ). 


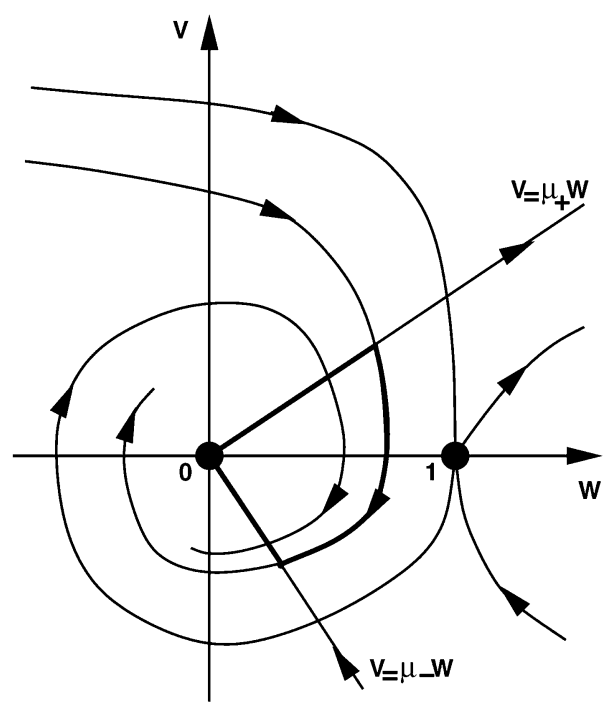

Fig. 3 Superimposed phase portraits of (10) and (11). The three pieces that are drawn in bold together form a sample trajectory that moves out of 0 along $v=\mu_{+} w$, next follows an orbit of (11), and finally moves back into 0 along $v=\mu_{-} w$.

Indeed, let us now prove this monotonicity property.

Lemma 2.1. Let $\left(v^{1}, w^{1}\right)$ and $\left(v^{2}, w^{2}\right)$ be two solutions of $(11)$ defined on $\left(a^{1}, b^{1}\right)$ and $\left(a^{2}, b^{2}\right)$, respectively, and satisfying

$$
v^{i}\left(a^{i}\right)=\mu_{+} w^{i}\left(a^{i}\right), \quad v^{i}\left(b^{i}\right)=\mu_{-} w^{i}\left(b^{i}\right)
$$

as well as

$$
\mu_{-}<\frac{v^{i}}{w^{i}}<\mu_{+} \quad \text { and } \quad w^{i}>0 \quad \text { in }\left(a^{i}, b^{i}\right)
$$

for $i=1,2$. Suppose that $v^{2}\left(a^{2}\right)>v^{1}\left(a^{1}\right)$. Then $b^{2}-a^{2}>b^{1}-a^{1}$.

Proof: By shifts of the solutions $w^{1}$ and $w^{2}$, (taking $w^{i}\left(x+a_{i}\right)$ ) there is no loss in generality in assuming that $a^{1}=a^{2}=0$. Then we want to show that $b^{2}>b^{1}$.

Recalling that $w_{x}=v$, Eq. (11) reads

$$
-\left(e^{c x} w_{x}\right)_{x}=e^{c x} w g(w),
$$

where $g(w)=r(1-w)$. From (13) and integration by parts, we see that for any $\alpha, 0<$ $\alpha<\operatorname{Min}\left\{b^{1}, b^{2}\right\}$, the following relation holds:

$$
\left[e^{-c x}\left(-w_{x}^{1} w^{2}+w_{x}^{2} w^{1}\right)\right]_{0}^{\alpha}=\int_{0}^{\alpha} e^{c x} w^{1} w^{2}\left(g\left(w^{1}\right)-g\left(x^{2}\right)\right) d x .
$$


Suppose that $w^{1}<w^{2}$ in $(0, \alpha)$ (which is certainly true for small $\left.\alpha>0\right)$. Then formula (14) shows that

$$
\frac{w_{x}^{2}(\alpha)}{w^{2}(\alpha)}>\frac{w_{x}^{1}(\alpha)}{w^{1}(\alpha)}
$$

Indeed, $g\left(w^{1}>g\left(w^{2}\right)\right.$ in $(0, \alpha)$. Now, if $\alpha<\min \left\{b^{1}, b^{2}\right\}$ is such that $w^{1}<w^{2}$ in $(0, \alpha)$ while $w^{1}(\alpha)=w^{2}(\alpha)$, then (15) yields $w_{x}^{2}(\alpha)>w_{x}^{1}(\alpha)$ which is impossible. Hence, $w^{1}$ and $w^{2}$ do not cross each other in $\left(0, \min \left\{b^{1}, b^{2}\right\}\right)$.

Assume now by way of contradiction that $b^{2} \leq b^{1}$. Then choosing $\alpha=b^{2}$ in (15), we get

$$
\frac{w_{x}^{1}(\alpha)}{w^{1}(\alpha)}<\mu^{-}
$$

again a contradiction. Therefore, $b^{2}>b^{1}$ and the lemma is proved.

So, the shortest feasible $L$ is obtained in the limit where the points on the line $v=\mu_{ \pm} w$ approach the origin. In that limit, we can replace the nonlinear term $-r w(1-w)$ in the second equation of (11) by its linearization $-r w$.

So, we want to connect the half-lines $v=\mu_{ \pm} w, w>0$, by a piece of orbit corresponding to

$$
\begin{aligned}
& w_{\xi}=v, \\
& v_{\xi}=-r w-c v,
\end{aligned}
$$

that is traversed in an interval of length $L$ or less. The general real solution of (16), for $\left(\frac{c}{2}\right)^{2}<r$, is given by

$$
\begin{aligned}
& w(\xi)=k \exp \left(\sigma_{+} \xi\right)+\bar{k} \exp \left(\sigma_{-} \xi\right), \\
& v(\xi)=\sigma_{+} k \exp \left(\sigma_{+} \xi\right)+\sigma_{-} \bar{k} \exp \left(\sigma_{-} \xi\right),
\end{aligned}
$$

where $k$ is an arbitrary complex number. If we require

$$
\begin{aligned}
& v(0)=\mu_{+} w(0), \\
& v(l)=\mu_{-} w(l),
\end{aligned}
$$

to determine the unknown $k$ and $l$, it seems that we have one real unknown too much, as $k$ counts for two. Note, however, that the system (18) is real homogeneous of degree one: If $k$ satisfies the equation, so does every real multiple of $k$. This reflects the fact that for the linear system (16), the "time" (i.e., the length of the independent variable interval) needed to cross the area between the lines $v=\mu_{ \pm} w$ is independent of the starting point on the line $v=\mu_{+} w$. So, we may add to (18) a condition that serves to normalize $k$. As such we choose

$$
\Re(k)=1 .
$$

The first equation of (18) then implies that

$$
\Im(k)=\frac{\Re\left(\sigma_{+}\right)-\mu_{+}}{\Im\left(\sigma_{+}\right)},
$$




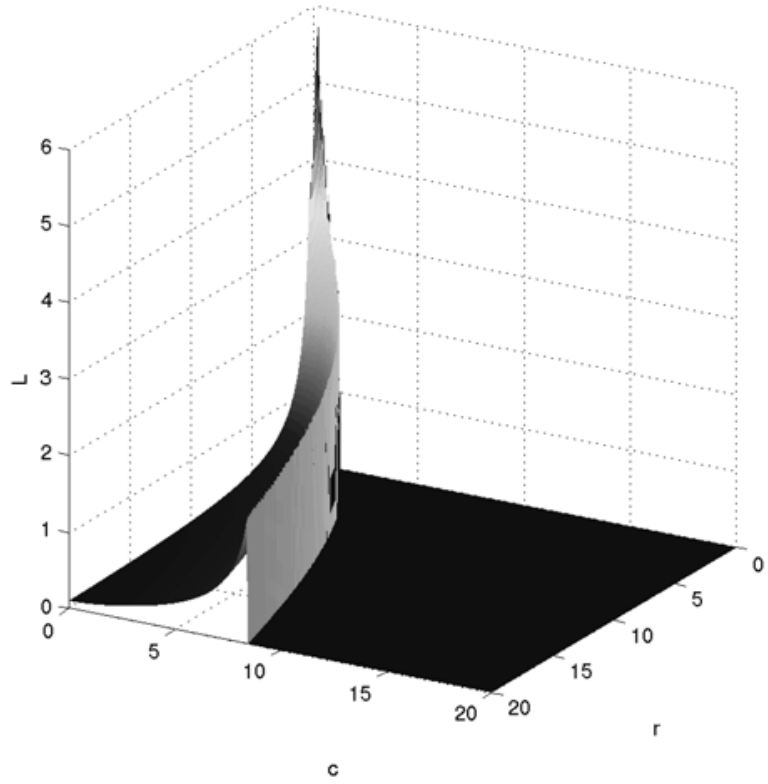

Fig. 4 Graphical representation of the condition (22) and the condition $c<2 \sqrt{r}$. A solution exists for parameter combinations in the domain "above" the depicted graph and to the left of the cylinder $c=2 \sqrt{r}$.

and now that $k$ is known, we can consider the second equation of (18) as determining $l$. After some manipulation, it can be rewritten as

$$
\begin{aligned}
& \tan \left(\Im\left(\sigma_{+}\right) l\right)=\frac{\left(\mu_{+}-\mu_{-}\right) \Im\left(\sigma_{+}\right)}{\mathfrak{R}^{2}\left(\sigma_{+}\right)+\mathfrak{\Im}^{2}\left(\sigma_{+}\right)-\mathfrak{R}\left(\sigma_{+}\right)\left(\mu_{-}+\mu_{+}\right)+\mu_{+} \mu_{-}}, \quad \text { or } \\
& \tan \left(\sqrt{r-\left(\frac{c}{2}\right)^{2}} l\right)=\frac{2 \sqrt{1+\left(\frac{c}{2}\right)^{2}} \sqrt{r-\left(\frac{c}{2}\right)^{2}}}{r-\frac{c^{2}}{2}-1} .
\end{aligned}
$$

Provided we adopt the convention that the function arctan takes its values in $(0, \pi]$ we can now formulate the conditions for the existence of a traveling wave solution as $c<2 \sqrt{r}$ and

$$
L>\frac{1}{\sqrt{r-\left(\frac{c}{2}\right)^{2}}} \arctan \left(\frac{2 \sqrt{1+\left(\frac{c}{2}\right)^{2}} \sqrt{r-\left(\frac{c}{2}\right)^{2}}}{r-\frac{c^{2}}{2}-1}\right) .
$$

These conditions are summarized in Fig. 4. Thus, for $c=\sqrt{2(r-1)}$ the right-hand side takes the value $\frac{\pi}{\sqrt{2(r+1)}}$, while for $c \uparrow 2 \sqrt{r}$ the right-hand side goes to infinity like $\frac{\pi}{\sqrt{r-\left(\frac{c}{2}\right)^{2}}}$. Using the scaling relations (7), we can rewrite (22) in terms of the original parameters as

$$
L>L_{\text {crit }},
$$


where

$$
L_{\text {crit }}=\frac{1}{\sqrt{\frac{r}{D}-\frac{c^{2}}{4 D^{2}}}} \arctan \left(\frac{2 \sqrt{\tilde{r}+\frac{c^{2}}{4 D}} \sqrt{r-\frac{c^{2}}{4 D}}}{r-\frac{c^{2}}{2 D}-\tilde{r}}\right),
$$

which should hold for $c<2 \sqrt{r D}$.

We shall rewrite the expression for $L_{\text {crit }}$ in a somewhat more informative form which, moreover, facilitates the comparison with the formula at the end of Section 4 in Potapov and Lewis (2004). To do so, we introduce

$$
c_{0}=2 \sqrt{r D}
$$

and recall that this so-called Fisher-speed is the asymptotic speed of propagation of disturbances (also called spreading or invasion speed) if all of the real line corresponds to favorable habitat (see Aronson and Weinberger, 1978). At the same time $c_{0}$ is the lowest speed for which for such a homogeneous favorable habitat, traveling wave solutions exist. The expression

$$
L_{\text {crit }}=\frac{\sqrt{\frac{D}{r}}}{\sqrt{1-\left(\frac{c}{c_{0}}\right)^{2}}} \arctan \left\{\frac{2 \sqrt{1-\left(\frac{c}{c_{0}}\right)^{2}} \sqrt{\frac{r}{\tilde{r}}+\left(\frac{c}{c_{0}}\right)^{2}}}{1-\frac{\tilde{r}}{r}-2\left(\frac{c}{c_{0}}\right)^{2}}\right\}
$$

has a factor $\sqrt{\frac{D}{r}}$ with the dimension of length, but is otherwise in terms of dimensionless quantities. The function arctan takes here values in $[0, \pi)$. Using the doubling formula

$$
\tan (2 \theta)=\frac{2 \tan (\theta)}{1-\tan ^{2}(\theta)}
$$

we can derive the alternative expression

$$
L_{\text {crit }}=\frac{2 \sqrt{\frac{D}{r}}}{\sqrt{1-\left(\frac{c}{c_{0}}\right)^{2}}} \arctan \left(\frac{\sqrt{\frac{\tilde{r}}{r}+\left(\frac{c}{c_{0}}\right)^{2}}}{\sqrt{1-\left(\frac{c}{c_{0}}\right)^{2}}}\right),
$$

where now arctan takes values in $\left[0, \frac{\pi}{2}\right)$. The corresponding expression in Potapov and Lewis (end of Section 4 ) has an extra factor $\sqrt{D}$ in the denominator of the argument of arctan, but is otherwise identical. We claim that this factor should not be there.

In the limit of an extremely hostile environment outside of the favorable patch, i.e., in the limit $\tilde{r} \rightarrow \infty$, we obtain the much simpler expression

$$
L_{\text {crit }}=\frac{\pi \sqrt{\frac{D}{r}}}{\sqrt{1-\left(\frac{c}{c_{0}}\right)^{2}}} .
$$

Formula (26) is related to the classical critical domain size problem when $c=0$ see Okubo and Levin (2001), Sections 9.1 and 10.2.2, and the references given therein. Indeed, the results there are recovered from ours by putting $c=0$. 


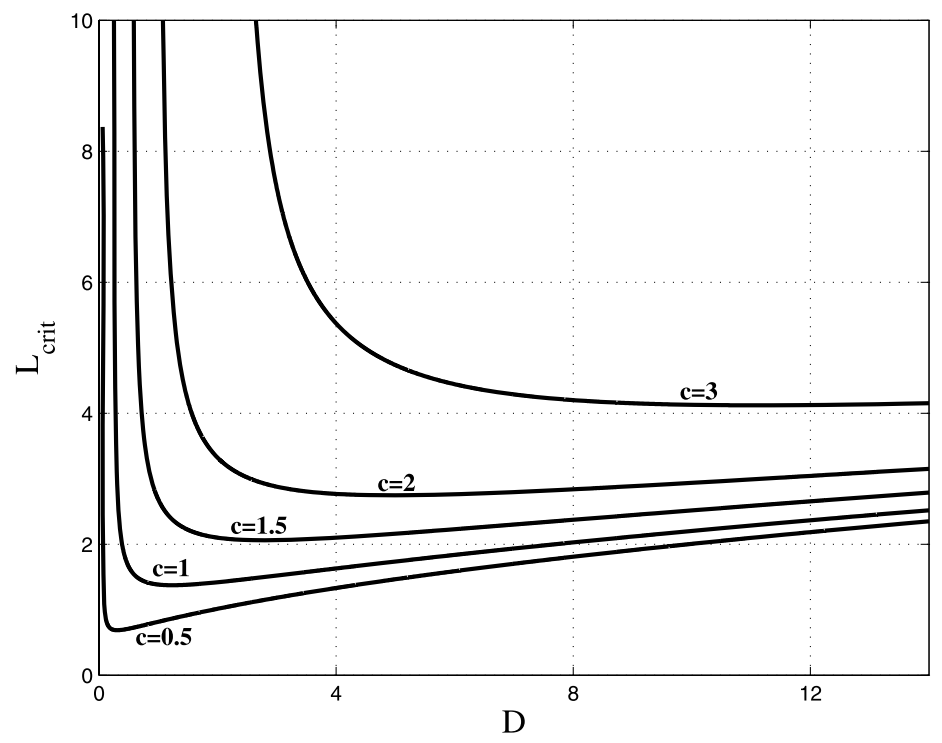

Fig. 5 Critical length $L(D, c)$, as a function of the diffusion coefficient $D$ for different values of the imposed speed $c$.

Note that here for $\frac{1}{2}<\left(\frac{c}{c_{0}}\right)^{2}<1$ the right-hand side of (26) decreases as a function of $D$, meaning that a species can survive on a smaller patch provided it increases its dispersal propensity, whereas for small $c$ it has to decrease D. (See also Fig. 5.) The reader should not be misled by the notation when verifying this statement: $c_{0}$ actually also depends on $D$. We can also rewrite the inequality $L>L_{\text {crit }}$ in the form

$$
\frac{r L^{2}}{2 \pi}-\sqrt{\frac{r^{2} L^{4}}{4 \pi^{4}}-\frac{c^{2} L^{2}}{4 \pi^{2}}}<D<\frac{r L^{2}}{2 \pi}+\sqrt{\frac{r^{2} L^{4}}{4 \pi^{4}}-\frac{c^{2} L^{2}}{4 \pi^{2}}} .
$$

From this it easily follows that in order to see persistence of the species, the diffusion constant should be neither too small nor too large, depending on $c$. This is illustrated in Fig. 5 which shows $L(D)$ achieving a minimum at a positive value of $D$, depending on $c$, for any $c>0$. Figure 6 displays the curve $L_{\text {crit }}(\tilde{r})$ for various values of $r$ with the asymptotic value as $\tilde{r} \rightarrow \infty$ given by formula (26) above. It further shows that for given $L, r$ has to have a minimum size for persistence.

Additional information can be obtained by computing the shape of the moving profile. Under our assumptions, the profile is symmetric when the climate does not move. In particular, there is no shape difference between the north and the south tail which are both maintained by migration from the favorable into the unfavorable area. The movement of the climate introduces asymmetry. As far as the tails are concerned, this is reflected in (9), which in terms of the original parameters and variables, implies that the spatio-temporal features of the tails are described by the expressions

$$
\mathrm{e}^{\left(-\frac{c}{2 D} \pm \sqrt{\left.\frac{\tilde{r}}{D}+\frac{c^{2}}{4 D^{2}}\right)(x-c t)}\right.} .
$$




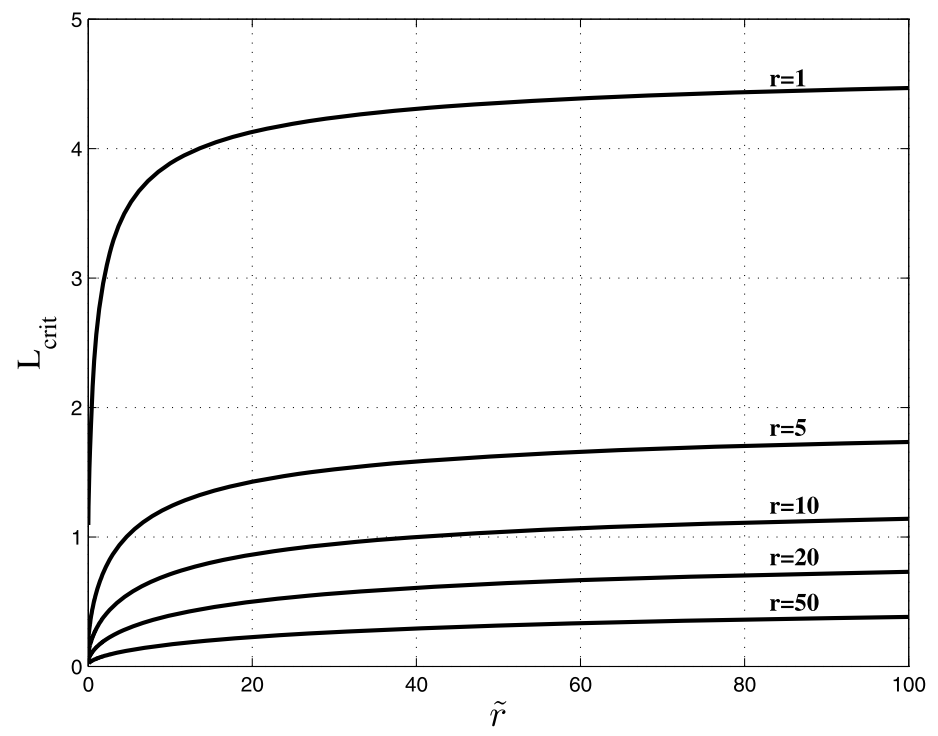

Fig. 6 Critical length $L=L(\tilde{r})$ for various values of $r$.

By analogy with $c_{0}$, the Fisher speed of invasion into the favorable habitat, we introduce a speed $\tilde{c}_{0}$ defined by the formula

$$
\tilde{c}_{0}=2 \sqrt{\tilde{r} D} \text {. }
$$

This speed can be thought of as representing the speed of retreat from the unfavorable region. Then we measure $c$ in terms of $\tilde{c}_{0}$ by putting

$$
c=\alpha \tilde{c}_{0}
$$

and write (27) in the form

$$
\mathrm{e}^{\left(-\alpha \pm \sqrt{1+\alpha^{2}}\right)\left(\sqrt{\frac{\tilde{r}}{D}} x-2 \alpha \tilde{r} t\right)}
$$

and conclude that the decay for positive $x$ is faster than the decay for negative $x$ by a factor

$$
\frac{\alpha+\sqrt{1+\alpha^{2}}}{-\alpha+\sqrt{1+\alpha^{2}}}
$$

Numerical results (see Fig. 7) show that when $\mathrm{c}$ is increased, the point at which the population achieves its maximum density shifts toward the south boundary of the patch. However, due to a tracking lag, it becomes closer to the north boundary of the population profile. Clearly this "body" effect strongly reinforces the asymmetry exhibited by the tails. Note that the steepness of the north front will make it relatively easy to determine from population census data that a shift took place and that in contrast, it may be much harder 

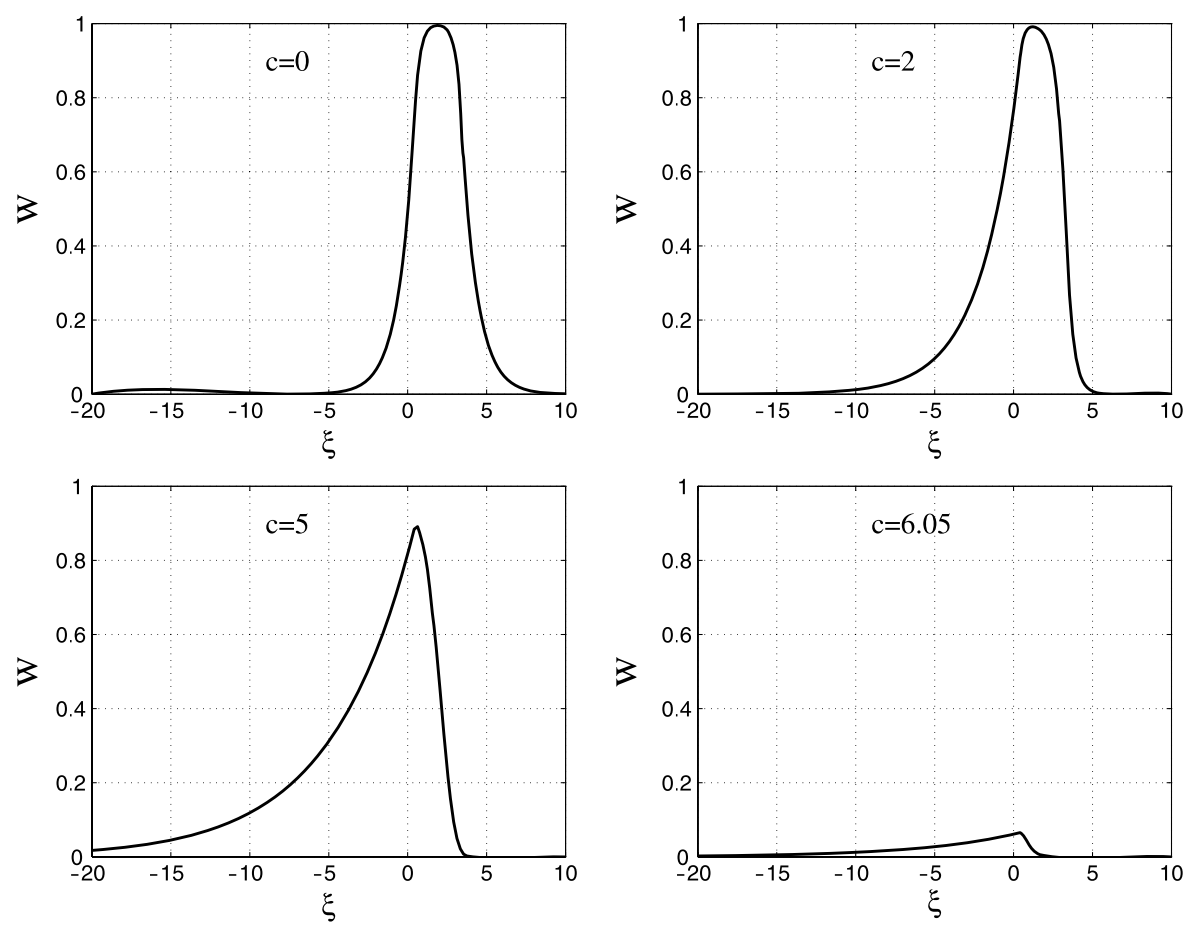

Fig. 7 Population profiles for various values of $c$, showing clearly that the north front can be a lot steeper than the south tail, that range size can both increase and decrease and that the point of maximum population density shifts toward the south boundary when $c$ increases. (The interval $[0,3]$ represents the patch of favorable habitat.)

to do so on the basis of a time series of observations in the south tail. This asymmetry is particularly visible on the two panels corresponding to the values $c=5$ and $c=6.05$ of Fig. 7. Parmesan et al. (1999) find exactly such a north-south asymmetry in a sample of 35 non-migratory European butterflies. They offer some speculations on possible causes. As explained above, our results provide a simple explanation on the basis of just the way in which the climate shift manifests itself in the (moving) population distribution. (See also the concluding remarks below.)

Another consequence of the asymmetry is that the range of the species may increase when the climate starts moving, when one defines the range as the spatial domain in which the population density exceeds a certain, somewhat arbitrarily chosen, lower bound (see Fig. 7). This phenomenon too derives from the relatively slow decay in the south tail. From Fig. 8, it is clear that the range keeps increasing until close to the critical speed, after which the range collapses fast. A distressing consequence of this threshold behavior is that a relatively small increase in climate speed can cause extinction with little advance warning.

The shape of the moving population profile is one aspect, total population size is another. A numerical "shooting" method to compute the total size of the "traveling" popu- 


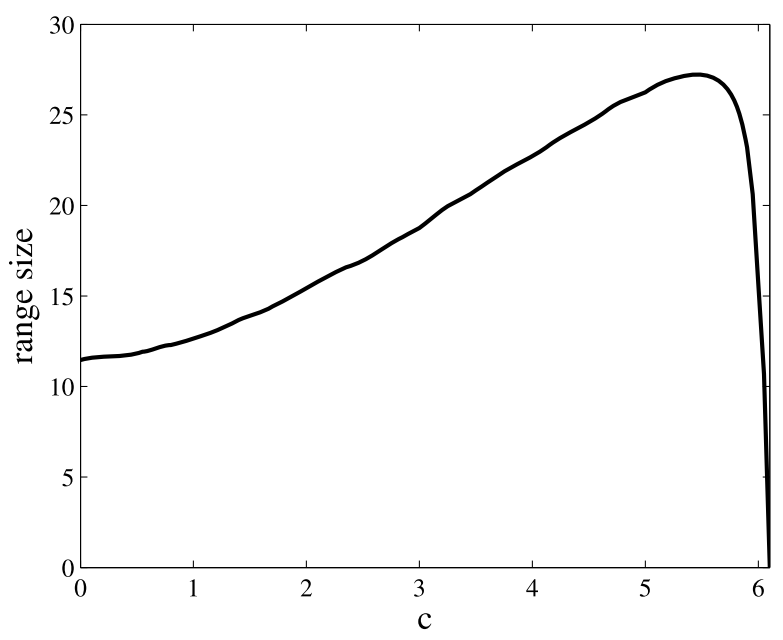

Fig. 8 Length of range $\{x ; w(x) \geq \varepsilon\}$ for $\varepsilon=0.01$.

lation as a function of the parameters is the following. Solve, for positive values of the parameter $Q$, the initial value problem

$$
\begin{aligned}
& \frac{d w}{d \xi}=v, \quad w(0)=Q, \\
& \frac{d v}{d \xi}=-r w(1-w)-c v, \quad v(0)=\mu_{+} Q
\end{aligned}
$$

up to $\xi=L$. If $\frac{v(L)}{w(L)}<\mu_{-}$, then $Q$ is too high. If $\frac{v(L)}{w(L)}>\mu_{-}$, then $Q$ is too low. By using a bisection-type technique, one can find an approximate solution for $Q$ to the equation $\frac{v(L)}{w(L)}=\mu_{-}$. In the last step of this iterative procedure, one adds to (32) the equation

$$
\frac{d N}{d \xi}=w, \quad N(0)=0 .
$$

The total population size then is given by

$$
N_{\text {tot }}=\frac{Q}{\mu_{+}}+N(L)-\frac{w(L)}{\mu_{-}},
$$

where the three contributions correspond to, respectively, the left tail, the middle part, and the right tail (see also Fig. 9).

The (counter intuitive) conclusion is that an increase in $c$ may lead to an increase of the total population size whenever the unsuitable area outside the favorable core area is not too harsh. This is due to a lag effect in the left tail: the decay of the population in the region that was favorable until recently may be slow while meanwhile, the rise of the population in the right region that just became favorable is relatively fast. This possibility of increases in both range and population size was not shown in the related work of Potapov and Lewis (2004). 

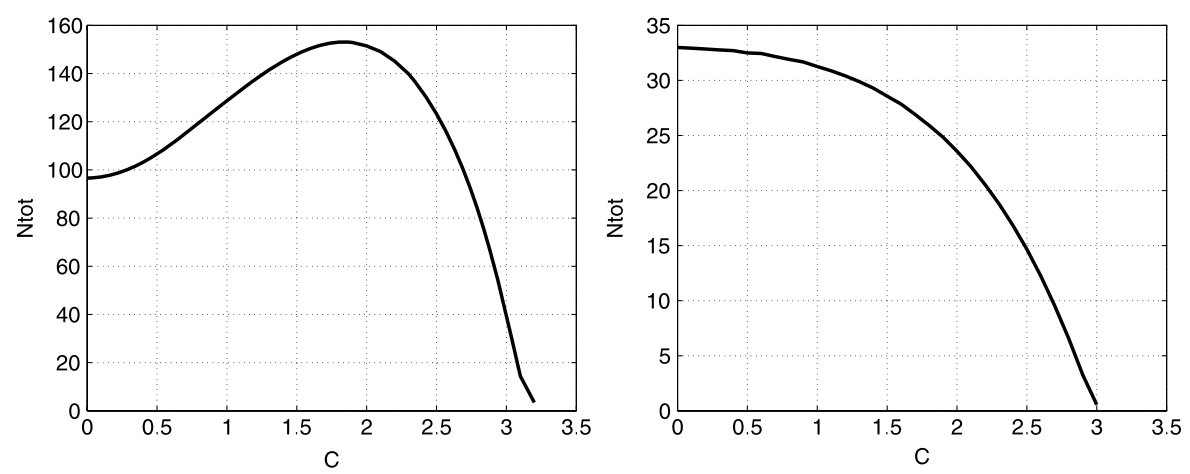

Fig. 9 Graphs of the total population size as a function of the speed $c$ at which the climate shifts. Left panel: $\tilde{r}=0.1$ and $L=3$; right panel: $\tilde{r}=1$ and $L=3$.

In conclusion of this section, we formulate an insight deriving from (23): for small $c$, an increase of $D$ entails an increase of the minimal interval length, since diffusion creates a net loss over the boundary of the favorable region. For larger $c$, however, the influence of $D$ on the minimal interval length may be opposite, since increased mobility helps to track the moving climate.

\section{The linearization at zero}

In this section, we investigate formally the stability of the extinct state. We find that the principal eigenvalue switches sign exactly at the codimension one manifold in parameter space that separates the domain of existence of a nontrivial solution from the domain of nonexistence. In the next section, we shall see that the principal eigenvalue for a general equation characterizes the existence and nonexistence of nontrivial solutions and determines as well the large time dynamics of this model. Note that within the domain of non-existence this eigenvalue further yields information about the rate of decay to zero, i.e., the rate at which the population declines on its way to extinction.

Returning to the general problem (1) with $f$ of the form (2), we note that the linearization at the trivial steady state $u \equiv 0$ is given by

$$
\frac{\partial u}{\partial t}=D \frac{\partial^{2} u}{\partial x^{2}}+g(0, x-c t) u
$$

To investigate the stability of $u \equiv 0$, we focus on solutions of (35) of the particular form

$$
u(x, t)=\mathrm{e}^{\lambda t} \phi(x-c t) .
$$

By substitution we deduce that such a solution exists if and only if $\phi$ is an eigenfunction corresponding to eigenvalue $\lambda$ for the linear differential operator $\mathcal{L}$ defined by

$$
(\mathcal{L} \phi)(\xi)=D \phi^{\prime \prime}(\xi)+c \phi^{\prime}(\xi)+g(0, \xi) \phi(\xi)
$$


In the next section, we will see that the sign of the principal (or dominant) eigenvalue of this operator, when properly defined, yields the long term dynamics in Eq. (1). Its sign gives a criterion for either extinction or persistence.

Therefore, methods to determine the sign of the dominant eigenvalue are of great interest, as are methods to give more quantitative estimates in case it is negative (at which time scale will the extinction happen?).

For the caricatural case of Section 2, we can derive an explicit equation for the dominant eigenvalue. The derivation follows the same pattern as the analysis leading to (22). In particular, we adopt the same scaling, which allows us to take $D=1$ in (37) and

$$
g(0, \xi)= \begin{cases}-1 & \xi<0 \text { and } \xi>L \\ r & 0 \leq \xi \leq L .\end{cases}
$$

Hence, the bounded solution of

$$
\mathcal{L} \phi=\lambda \phi,
$$

which is normalized by the condition

$$
\phi(0)=1,
$$

is given by

$$
\phi(\xi)=\mathrm{e}^{\left(-\frac{c}{2}+\sqrt{\left.\left(\frac{c}{2}\right)^{2}+\lambda+1\right)} \xi\right.},
$$

for $\xi<0$ whenever the expression under the square root is positive. For $0 \leq \xi \leq L$, on the other hand, the solution is represented by

$$
\phi(\xi)=k \mathrm{e}^{\left(-\frac{c}{2}+\sqrt{\left.\left(\frac{c}{2}\right)^{2}+\lambda-r\right) \xi}\right.}+\bar{k} \mathrm{e}^{\left(-\frac{c}{2}-\sqrt{\left.\left(\frac{c}{2}\right)^{2}+\lambda-r\right) \xi}\right.},
$$

where $k$ is a complex number and, by assumption, the expression under the square root is now negative. Finally, for $\xi>L$, we should have

$$
\phi(\xi)=C \mathrm{e}^{\left(-\frac{c}{2}-\sqrt{\left(\frac{c}{2}\right)^{2}+\lambda+1}\right) \xi} .
$$

It remains to determine $k$ and $C$ from linking conditions that should guarantee that $\phi$ is continuously differentiable at both $\xi=0$ and $\xi=L$. From the smoothness condition at $\xi=0$, we deduce

$$
k=\frac{1}{2}-i \frac{\sqrt{\left(\frac{c}{2}\right)^{2}+\lambda+1}}{2 \sqrt{r-\lambda-\left(\frac{c}{2}\right)^{2}}} .
$$

Eliminating $C$ from the smoothness condition at $\xi=L$, we end up with one equation for the unknown $\lambda$. This equation is the analogue of (21). It reads

$$
\tan \left\{\sqrt{r-\lambda-\left(\frac{c}{2}\right)^{2}} L\right\}=\frac{2 \sqrt{1+\lambda+\left(\frac{c}{2}\right)^{2}} \sqrt{r-\lambda-\left(\frac{c}{2}\right)^{2}}}{r-2 \lambda-\frac{c^{2}}{2}-1} .
$$


As a consequence of the more general results in the next section, it can be shown, that the condition $\lambda=0$ in (45) is equivalent to the critical length condition of the previous section.

Note that $\lambda$ and $c$ only occur in the combination $\lambda+\left(\frac{c}{2}\right)^{2}$. In terms of the unscaled time and parameters, this means that

$$
\lambda(c)=\lambda(0)-\frac{c^{2}}{4 D} .
$$

In other words, the dominant eigenvalue is a quadratically decreasing function of $c$, with a coefficient of the quadratic term which is inversely proportional to $D$ but independent of all other parameters. The relation (46) can be derived by a Liouville transformation $\phi(x)=\exp \left(-\frac{c}{2 D} x\right) \psi(x)$ which eliminates the first order derivative from the eigenvalue problem $\mathcal{L} \phi=\lambda \phi$. So, it holds for general functions $g(0, \xi)$, not just for (38).

\section{Analysis of a general class of equations}

So far, we have considered a particular type of heterogeneity, that which is obtained by juxtaposing two homogeneous media-the favorable and unfavorable ones-with an abrupt transition at the two end points of the favorable interval. Are the results which we have derived previously robust? And is the co-moving nontrivial solution stable if it exists? Here we give very strong affirmative answers to both these questions in a rather general setting. The motivation for considering general types of nonlinearities is twofold. First, the assumptions made in Section 2 are rather contrived from a modeling point of view and one would like to consider more complex transitions e.g. gradual transitions between recognizable but not necessarily strictly homogeneous zones. Second, a general mathematical theory sheds much more light on the underlying mechanisms, since the proofs reveal the role that various assumptions play in yielding the conclusions. Here, for instance, the linearization at the trivial steady state, in particular the sign of the associated principal eigenvalue, will be seen to fully account for the ability to keep pace with a shifting climate.

In this section, we consider Eq. (1). As was already mentioned, there is no loss in generality in assuming that $D=1$, which we do henceforth.

The functions $f$ and $g$ (related through (2)) will be assumed to satisfy the following set of conditions.

(a) Negative density dependence: $u \mapsto g(u, x)$ is decreasing for all $x \in \mathbb{R}$ and strictly decreasing for $x \in I_{0}$, where $I_{0}$ is a nonempty open interval.

(b) Allow for multiple discontinuities, e.g., several patches: there is a finite set of points $F=\left\{a_{1}, \ldots, a_{p}\right\}$ in $\mathbb{R}$ such that $g$ is continuous on $\mathbb{R}_{+} \times(\mathbb{R} \backslash F)$ and both $\lim _{x \uparrow a_{i}} g(u, x)$ and $\lim _{x \downarrow a_{i}} g(u, x)$ exist, uniformly for $u$ in compact subsets of $\mathbb{R}_{+}$.

(c) Existence of a linearization: There exists $\delta>0$ such that $u \mapsto g(u, x)$ is $C^{1}$ on $[0, \delta]$ for all $x \in \mathbb{R}, g_{u}$ is continuous on $[0, \delta] \times(\mathbb{R} \backslash F)$ and both $\lim _{x \uparrow a_{i}} g_{u}(u, x)$ and $\lim _{x \downarrow a_{i}} g_{u}(u, x)$ exist, uniformly for $u \in[0, \delta]$.

(d) Unfavorable outer regions: $g(0, x) \rightarrow-1$ as $x \rightarrow \pm \infty$.

(e) Saturation: There exists $M>0$ such that $g(u, x) \leq 0$ for all $x \in \mathbb{R}$ whenever $u \geq M$. 
The properties formulated in (a)-(e) above are the standing hypotheses on the function $g$ throughout this section. The last one means that everywhere the population declines when it exceeds some level M, i.e., negative density dependence guarantees that the population stays bounded. The limits at $\pm \infty$ in (d) are taken to be the same in order to simplify the formulation, but the statements and proofs can readily be adapted to the case of different limits. Note that the values of the limit can be changed by a scaling of the time variable $t$. Accordingly, the value -1 is representative for general negative values. Similarly it is no restriction that we take $D=1$, as this can always be achieved by a scaling of the spatial variable $x$ (after the scaling of time). Since $g$ may have discontinuities with respect to $x$, we consider generalized solutions. These are functions $u$ which, as a function of $x$, are globally of class $C^{1}$ and piecewise of class $C^{2}$ and satisfy the equation at each point with $x \neq a_{i}, i=1, \ldots, p$.

For studies of solutions of (1) on bounded domains, without an imposed translation speed (i.e., $c=0$ ), we refer to Murray and Sperb (1983), Cantrell and Cosner (1991, 1998, 2003), Cano-Casanova and López-Gómez (2003) and the references given there. Recently, the effect of a heterogeneous but spatially periodic environment has been studied by Berestycki et al. (2005a, 2005b). Lastly, periodic stochastic environments are considered by Roques and Stoica (2007).

The problem we study here involves a lack of compactness (the problem is set on the whole real line) as well as the difficulty deriving from the fact that $c$ is imposed.

Our first aim is to give necessary and sufficient conditions for the existence of a traveling wave solution, that is, of a bounded solution $w>0$ of (5). We shall find that such a solution exists if and only if the zero steady state of the equation

$$
\frac{\partial u}{\partial t}=\frac{\partial^{2} u}{\partial \xi^{2}}+c \frac{\partial u}{\partial \xi}+f(u, \xi)
$$

(which is just (1) rewritten in terms of a moving coordinate system) is linearly unstable in the sense that an associated dominant eigenvalue is positive. Next, we settle the uniqueness issue by showing that there is at most one traveling wave solution. Concerning the large time asymptotic behavior of solutions of the initial value problem for (1), we then formulate a dichotomy:

- If no traveling wave solution exists, every positive solution of (1) converges to zero for $t \rightarrow \infty$, uniformly in $x$.

- If a traveling wave solution $w$ exists, every nontrivial positive solution $u(t, x)$ of (1) converges for $t \rightarrow \infty$ to $w(x-c t)$, uniformly in $x$.

\subsection{A priori estimates for the "far out" asymptotic behavior of traveling waves}

We will replace the symbol $\xi$ by the symbol $x$ in order to facilitate the reference to the literature. Thus, we write (5) with $D=1$ as

$$
w_{x x}+c w_{x}+w g(w, x)=0 .
$$

We start by analyzing the limiting behavior as $x \rightarrow \pm \infty$ that any bounded positive solution necessarily has. Indeed, note that no conditions at infinity, other than being bounded are imposed here on solutions. 
Recall that the quantities $\mu_{ \pm}$are defined in (9) and that they are the roots of $\lambda^{2}+c \lambda-$ $1=0$. It is tempting to conjecture that for some positive constants $a, b, w(x) \sim a \mathrm{e}^{\mu+x}$ for $x \rightarrow-\infty$ and $w(x) \sim b \mathrm{e}^{\mu_{-} x}$ for $x \rightarrow+\infty$. This is indeed true if, for instance, $g(0, x)=$ -1 for large $|x|$ as was the case in Sections 2 and 3 above. (More general results in this direction can be found in Berestycki and Nirenberg, 1991.) But, in general, it is not so. Indeed, if $g(0, x)$ converges slowly to -1 we do not, in general, obtain exact exponential behavior. For instance,

$$
w(x)=(1+x) \mathrm{e}^{-(1+\sqrt{2}) x}
$$

is a solution on $\mathbb{R}_{+}$of the equation

$$
w_{x x}+c w_{x}+h(x) w=0
$$

with

$$
h(x)=-1+\frac{2 \sqrt{2}}{1+x} .
$$

This observation motivates us to formulate that $w(x)$ behaves like $\mathrm{e}^{\mu+x}$ for $x \rightarrow-\infty$ and like $\mathrm{e}^{\mu_{-} x}$ for $x \rightarrow+\infty$ in a weaker sense that we now make precise.

Proposition 4.1. Let $w$ be a bounded positive solution of (48). Then $w(x) \rightarrow 0$ for $x \rightarrow$ $\pm \infty$. In fact, for any $\varepsilon>0$

$$
w(x) \mathrm{e}^{\left(-\mu_{-}-\varepsilon\right) x} \rightarrow 0 \quad \text { for } x \rightarrow \infty
$$

and

$$
w(x) \mathrm{e}^{\left(-\mu_{+}+\varepsilon\right) x} \rightarrow 0 \quad \text { for } x \rightarrow-\infty .
$$

Proof: We start by proving that $w(x) \rightarrow 0$ for $x \rightarrow \infty$. There exists $R>0$ such that for all $x \geq R$ the inequality

$$
g(0, x) \leq-v
$$

holds for, say, $v=\frac{1}{2}$. Hence, (a) implies that for $x>R$,

$$
w_{x x}+c w_{x}-v w \geq 0
$$

and, by the maximum principle, it follows that for all $a>0$, for $x \in(R, R+a)$ the inequality

$$
w \leq \psi^{a}
$$

holds where $\psi^{a}$ is defined by the boundary value problem

$$
\left\{\begin{array}{l}
\psi_{x x}^{a}+c \psi_{x}^{a}-v \psi^{a}=0, \quad R<x<R+a, \\
\psi^{a}(R)=M=\psi^{a}(R+a)
\end{array}\right.
$$


with

$$
M:=\sup w .
$$

It should be noted here, also for future use, that even though $f$ may be discontinuous, the maximum principle still applies to the $C^{1}$ solutions that we consider. In the present one-dimensional setting, this can be verified rather directly. More general statements in Gilbarg and Trudinger (1998) also cover the multi-dimensional situation.

A direct computation shows that

$$
\psi^{a}(x)=M\left(\frac{1-\mathrm{e}^{\rho_{-} a}}{\mathrm{e}^{\rho_{+} a}-\mathrm{e}^{\rho_{-} a}}\right) \mathrm{e}^{\rho_{+}(x-R)}+M\left(\frac{\mathrm{e}^{\rho_{+} a}-1}{\mathrm{e}^{\rho_{+} a}-\mathrm{e}^{\rho_{-} a}}\right) \mathrm{e}^{\rho_{-}(x-R)},
$$

where

$$
\begin{aligned}
& \rho_{+}=\frac{-c+\sqrt{c^{2}+4 v}}{2}, \\
& \rho_{-}=\frac{-c-\sqrt{c^{2}+4 v}}{2}
\end{aligned}
$$

(i.e., $\rho_{ \pm}$are the roots of $r^{2}+c r-v=0$ with $\rho_{-}<0<\rho_{+}$). Since

$$
\psi^{a}(x) \rightarrow M \mathrm{e}^{\rho_{-}(x-R)} \quad \text { for } a \rightarrow \infty
$$

we obtain, by taking the limit $a \rightarrow \infty$, the inequality

$$
w(x) \leq M \mathrm{e}^{\rho_{-}(x-R)} \quad \text { for } x \geq R
$$

which shows that $w(x) \rightarrow 0$ for $x \rightarrow \infty$.

Similarly, one derives for some $R$ the inequality

$$
w(x) \leq M \mathrm{e}^{\rho_{+}(x+R)} \quad \text { for } x \leq-R
$$

and concludes from this that $w(x) \rightarrow 0$ for $x \rightarrow-\infty$.

Next, in order to derive the more precise description of the limiting behavior of $w$ given by (49) and (50), we first observe that in the previous argument $v$ should be less than 1 , but can otherwise be chosen as close to 1 as we wish: For all $v$ with $0<v<1$, there exists $R=R(v)$ such that $g(0, x) \leq-v$ for $x \geq R$. Since $\rho_{ \pm} \rightarrow \mu_{ \pm}$as $v \uparrow 1$, it follows from (50) and (51) that for all $\delta>0$ there exists $R=R(\delta)>0$ such that

$$
\begin{array}{ll}
w(x) \leq M \mathrm{e}^{\left(\mu_{-}+\delta\right) x} & \text { for } x \geq R, \\
w(x) \leq M \mathrm{e}^{\left(\mu_{+}-\delta\right) x} & \text { for } x \leq-R .
\end{array}
$$

Next, we want to derive lower bounds. We first formulate an auxiliary result that will also be used later.

Proposition 4.2. Let $v: \mathbb{R} \rightarrow \mathbb{R}$ be positive, piecewise $C^{2}$ and such that for all but at most finitely many, $x$ in an interval of the form $(R, \infty)$ the inequality

$$
v_{x x}+c v_{x}-v v \leq 0
$$


holds, where $c$ and $v$ are such that $\left(\frac{c}{2}\right)^{2}+v>0$. Then there exists a positive constant $K$ such that

$$
v(x) \geq K \mathrm{e}^{\left(-\frac{c}{2}-\sqrt{\left.\left(\frac{c}{2}\right)^{2}+v\right)} x\right.} \quad \text { for } x \geq R .
$$

If, similarly, the differential inequality holds for $x$ in an interval of the form $(-\infty,-R)$ then there exists a positive constant $K$ such that

$$
v(x) \geq K \mathrm{e}^{\left(-\frac{c}{2}+\sqrt{\left.\left(\frac{c}{2}\right)^{2}+v\right)} x\right.} \quad \text { for } x \leq-R .
$$

We omit the (easy) proof, since it follows exactly the same line of argumentation that we used to prove the preceding proposition. Now let $w$ be a bounded positive solution of (48). Then since $w(x) \rightarrow 0$ for $x \rightarrow \pm \infty$, for every $\delta>0$, there exists a $R=R(\delta)$ such that

$$
w_{x x}+c w_{x}-(1+\delta) w \leq 0
$$

for $x \geq R$ and for $x \leq-R$. Thus, as a corollary of Proposition 4.2, we obtain the following proposition.

Proposition 4.3. Let $w$ be a bounded positive solution of (48). Then for any $\varepsilon>0$, we have that

$$
w(x) \mathrm{e}^{\left(-\mu_{-}+\varepsilon\right) x} \rightarrow \infty \quad \text { for } x \rightarrow \infty
$$

and

$$
w(x) \mathrm{e}^{\left(-\mu_{+}-\varepsilon\right) x} \rightarrow \infty \quad \text { for } x \rightarrow-\infty .
$$

To conclude this subsection, we formulate an estimate for the derivative of $w$.

Proposition 4.4. Let $w$ be a bounded positive solution of (48). For every $\varepsilon>0$, there exists $R=R(\varepsilon)>0$ such that

$$
\begin{array}{ll}
\left|w_{x}(x)\right| \leq \mathrm{e}^{\left(\mu_{-}+\varepsilon\right) x} & \text { for } x \geq R, \\
\left|w_{x}(x)\right| \leq \mathrm{e}^{\left(\mu_{+}-\varepsilon\right) x} & \text { for } x \leq-R .
\end{array}
$$

Proof: We restrict our attention to large positive $x$, the case of negative $x$ being the same. From (48), we get

$$
w_{x}(z)-w_{x}(y)+c w(z)-c w(y)=-\int_{y}^{z} w(x) g(w(x), x) d x .
$$

This shows that the limit of $w_{x}(z)$ exists when $z \rightarrow \infty$, hence that $w_{x}(\infty)=0$. Therefore, letting $y \rightarrow \infty$ in this relation yields the identity

$$
w_{x}(x)+c w(x)+\int_{\infty}^{x} w(y) g(w(y), y) d y=0 .
$$

The result now follows from the properties of $g$ and the estimates for $w$ obtained in Proposition 4.1. 


\subsection{The eigenvalue problem}

As another preparatory step, we shall make precise how, in the present case, the principal (or dominant) eigenvalue of the linearized problem at $w \equiv 0$ is defined. Since we consider solutions defined on the whole real line, some special care is needed.

Let $L_{R}$ denote the differential operator:

$$
L_{R} \phi=\phi_{x x}+c \phi_{x}+g(0, \cdot) \phi .
$$

Let $\lambda_{R}$ denote the principal eigenvalue of $L_{R}$ associated with zero Dirichlet boundary conditions

$$
\phi(-R)=0=\phi(R) .
$$

Then the corresponding eigenfunction $\phi^{R}$ is strictly positive on $(-R, R)$. It is well known that $R \mapsto \lambda_{R}$ is increasing (see, for instance Berestycki et al., 1994 for a monotonicity proof in a more general framework). So, it is meaningful to formulate the following definition.

\section{Definition 4.5.}

$$
\lambda_{\infty}:=\lim _{R \rightarrow \infty} \lambda_{R}
$$

There are alternative ways to define $\lambda_{\infty}$, see Berestycki et al. (1994), Pinsky (1995), or Berestycki et al. (2007) for a formula that applies to general operators in unbounded domains. As a special case of the results established in Berestycki et al. (1994), we obtain the characterization

$$
\begin{array}{r}
\lambda_{\infty}=\sup \left\{t \in \mathbb{R}: \exists \phi \in W_{\text {loc }}^{2, \infty}(\mathbb{R}) \text { such that } \phi>0\right. \\
\text { and } \left.\phi^{\prime \prime}+c \phi^{\prime}+g(0, \cdot) \phi+t \phi \leq 0 \text { on } \mathbb{R}\right\} .
\end{array}
$$

Note that the $\phi$ that we consider here are allowed to grow beyond any bound for $|x| \rightarrow \infty$. By restricting the "test" functions $\phi$ to those that are bounded on $\mathbb{R}$, we obtain a different generalized eigenvalue that may be smaller. For instance, if $g(0, x)=-1$ for all $x$, then $\lambda_{\infty}=1+\frac{c^{2}}{4}$ while the additional requirement that the functions be bounded yields a generalized eigenvalue equal to 1 ; so when $c \neq 0$, these are not equal. We refer to Berestycki et al. (2007) for a general study of these themes.

In the current context, the motivation to call $\lambda_{\infty}$ the principal eigenvalue derives from the results presented in the next subsection and in Section 4.5. In the proof, we shall need that a positive $\phi^{\infty} \in W_{\text {loc }}^{2, \infty}$ exists such that

$$
\phi_{x x}^{\infty}+c \phi_{x}^{\infty}+g(0, \cdot) \phi^{\infty}=\lambda_{\infty} \phi^{\infty}
$$

on $\mathbb{R} \backslash F$. Such a $\phi^{\infty}$ is obtained as the limit, uniformly on compact subsets, of a sequence $\phi^{R_{j}}$ for some sequence $R_{j}$ such that $R_{j} \rightarrow \infty$ as $j \rightarrow \infty$. The idea is to first normalize $\phi^{R}$ by requiring that for instance, $\phi^{R}(0)=1$. Next, we invoke the Harnack inequality (see Ladyženskaja et al., 1968, Section III.10, p. 209 or Krylov, 1987, Section 4.2, p. 130), 
stating that on a given bounded interval $(-A, A)$ and for $R$ sufficiently large, the maximum of $\phi^{R}$ is bounded by a constant (depending on $A$, but not on $R$ ) times the minimum of $\phi^{R}$. Since the minimum of $\phi^{R}$ is bounded by 1 , we obtain an $R$ independent bound on the maximum of $\phi^{R}$. The regularity theory of elliptic equations next guarantees that any sequence has a converging subsequence and that we can pass, for such a subsequence, to the limit in the differential equation.

We conclude this subsection by describing, in a crude manner, the "far out" asymptotic behavior of $\phi^{\infty}$ when $\lambda_{\infty}$ is either negative or zero. We only state the properties that we shall use in the next subsection.

Proposition 4.6. Let $\phi^{\infty}$ be positive and satisfy (56) on $\mathbb{R} \backslash F$ with $\lambda_{\infty}<0$. For every $\delta$ with $\max \left\{0,-1-\lambda_{\infty}\right\}<\delta$, there exist $R(\delta)$ and $K(\delta)$ such that

$$
\begin{array}{ll}
\phi(x) \geq K \mathrm{e}^{\left(-\frac{c}{2}-\sqrt{\left(\frac{c}{2}\right)^{2}+1+\delta+\lambda_{\infty}}\right) x} & \text { for } x \geq R, \\
\phi(x) \geq K \mathrm{e}^{\left(-\frac{c}{2}+\sqrt{\left(\frac{c}{2}\right)^{2}+1+\delta+\lambda_{\infty}}\right) x} & \text { for } x \leq-R .
\end{array}
$$

Proof: Again, we restrict our attention to large positive $x$. For any $\delta>0$, the function $\phi^{\infty}$ satisfies for large enough $x$ the differential inequality

$$
\phi_{x x}+c \phi_{x}-\left(1+\delta+\lambda_{\infty}\right) \phi \leq 0 .
$$

So the inequality (57) follows from Proposition 4.2, provided the argument of the squareroot is positive. This requires that $\delta>-1-\lambda_{\infty}$ and since we already required that $\delta>0$, we should restrict to $\delta>\max \left\{0,-1-\lambda_{\infty}\right\}$.

Proposition 4.7. Let $\phi^{\infty}$ be positive and satisfy (56) on $\mathbb{R} \backslash F$ with $\lambda_{\infty}=0$. Then $\phi^{\infty}$ has the properties formulated in terms of $w$ as (49), (50), and in Proposition 4.4.

Sketch of the proof: The proof is essentially identical to the proofs of Propositions 4.1 and 4.4. Here, however, we do not a priori know that $\phi^{\infty}$ is bounded. The idea is to replace the $\psi^{a}$ from the proof of Proposition 4.1 by functions $z^{R}$ which satisfy

$$
\left\{\begin{array}{l}
z_{x x}+c z_{x}-v z=0, \quad p<x<R \\
z(p)=\alpha, \quad z(R)=0
\end{array}\right.
$$

where $p$ is such that $g(0, x) \leq-v$ for $x>p$ and $\alpha:=\sup _{R} \phi^{R}(p)$. One then combines the inequality $\phi^{R}(x) \leq z^{R}(x)$, for $x \in(p, R)$, with the fact that for $R \rightarrow \infty$

$$
z^{R}(x) \rightarrow \alpha \mathrm{e}^{\rho_{-}(x-p)} \quad \text { for } x \geq p .
$$

\subsection{The solvability condition}

Theorem 4.8. Equation (48) has a bounded positive solution if and only if $\lambda_{\infty}>0$.

Proof: Assume that $\lambda_{\infty}>0$. We shall prove that a solution exists by constructing both a sub- and a supersolution. Recall that $\lambda_{R}$ denotes the principal eigenvalue of $L_{R}$ defined 
by (53)-(54). Again, we denote by $\phi^{R}$ the associated positive eigenfunction, but this time we normalize by requiring that the maximum of $\phi^{R}$ equals one. Now define

$$
v(x)= \begin{cases}\varepsilon \phi^{R}(x) & \text { for }-R \leq x \leq R, \\ 0 & \text { for }|x| \geq R .\end{cases}
$$

Then for $-R<x<R$,

$$
v_{x x}(x)+c v_{x}(x)+g(v(x), x) v(x)=[g(v(x), x)-g(0, x)] v(x)+\lambda_{R} v(x) .
$$

We claim that for $R$ large enough and for $\varepsilon>0$ small enough, $v$ is a subsolution, i.e., the right-hand side is positive. To substantiate the claim, we first note that $\lambda_{R}>0$ for large $R$ since $\lambda_{R} \rightarrow \lambda_{\infty}$ for $R \rightarrow \infty$ and $\lambda_{\infty}>0$. Next, observe that $g(v(x), x)-g(0, x) \rightarrow 0$ for $\varepsilon \downarrow 0$. Lastly, it is known, that since $\phi^{R}( \pm R)=0$, extending $\varepsilon \phi^{R}$ by 0 outside $(-R, R)$ yields a subsolution $v$.

Assumption (e) guarantees that the constant function taking the value $M$ is a supersolution. Clearly, $v(x)<M$ for small $\varepsilon$. We conclude that a solution exists.

It remains to verify the necessity of the condition $\lambda_{\infty}>0$. We assume that a bounded positive solution $w$ of (48) exists and that $\lambda_{\infty} \leq 0$ and then try to reach a contradiction. We start by making the stronger assumption $\lambda_{\infty}<0$. Let $\phi^{\infty}$ be positive and satisfy (56) on $\mathbb{R} \backslash F$. We claim that

$$
\lim _{x \rightarrow \pm \infty} \frac{\phi^{\infty}(x)}{w(x)}=\infty .
$$

Indeed, this follows by combining (57) with (48) and (58) with (49), if we choose $\delta$ in Proposition 4.6 such that not only $\delta>\max \left\{0,-1-\lambda_{\infty}\right\}$ but also $\delta<-\lambda_{\infty}$. The point is that in this case $\sqrt{\left(\frac{c}{2}\right)^{2}+1+\delta+\lambda_{\infty}}<\sqrt{\left(\frac{c}{2}\right)^{2}+1}$ so that by choosing next $\varepsilon$ in Proposition 4.1 sufficiently small, the quotient $\phi^{\infty}(x) / w(x)$ has a positive exponent for large positive $x$ and a negative exponent for large negative $x$.

Since $\phi^{\infty}>0, w>0$, and for large $|x|$, the function $w$ is "dominated" by $\phi^{\infty}$, the set

$$
\left\{\alpha: \alpha \phi^{\infty} \geq w \text { on } \mathbb{R}\right\}
$$

is nonempty. Let $\alpha_{0}$ be the infimum of this set. Then $\alpha_{0}>0$ and by continuity, $\alpha_{0} \phi^{\infty} \geq w$ on $\mathbb{R}$, i.e., $\alpha_{0}$ belongs to the set. Since $\phi^{\infty}(x) / w(x) \rightarrow \infty$ for $|x| \rightarrow \infty$, there exists $R>0$ such that $\alpha \phi^{\infty} \geq w$ for $|x| \geq R$ and $\frac{1}{2} \alpha_{0}<\alpha<\alpha_{0}$. If $\min \left\{\alpha_{0} \phi^{\infty}(x)-w(x)\right.$ : $-R \leq x \leq R\}$ would be positive, we arrive at a contradiction with the definition of $\alpha_{0}$. So, this minimum must be zero, i.e., the positive function $v:=\alpha_{0} \phi^{\infty}-w$ assumes its minimum value zero. Since

$$
v_{x x}+c v_{x}+g(0, \cdot) v=(g(w(\cdot), \cdot)-g(0, \cdot)) w+\lambda_{\infty} \alpha_{0} \phi^{\infty}
$$

and the right-hand side of this identity is nonpositive, the strong maximum principle states that this is only possible if $v \equiv 0$, which is clearly impossible. So, $\lambda_{\infty}<0$ precludes the existence of $w$.

Now assume that $\lambda_{\infty}=0$. We rewrite the equation for $\phi^{\infty}$ in self-adjoint form as

$$
\left(\mathrm{e}^{c x} \phi_{x}^{\infty}\right)_{x}+g(0, x) \mathrm{e}^{c x} \phi^{\infty}=0 .
$$


The analogue form of the equation for $w$ reads

$$
\left(\mathrm{e}^{c x} w_{x}\right)_{x}+g(w(x), x) \mathrm{e}^{c x} w=0 .
$$

If we multiply the equation for $w$ by $\phi^{\infty}$, the equation for $\phi^{\infty}$ by $w$, integrate by parts over $[-A, A]$ and then subtract, we obtain the identity

$$
\left[-\mathrm{e}^{c x} \phi_{x}^{\infty} w+\mathrm{e}^{c x} w_{x} \phi^{\infty}\right]_{x=-A}^{x=+A}=\int_{-A}^{A}(g(0, x)-g(w(x), x)) \mathrm{e}^{c x} \phi^{\infty}(x) w(x) d x .
$$

Now $g(0, x)-g(w(x), x) \geq 0$ for all $x$, but with strict inequality for $x \in I_{0}$ (compare condition (4.1)). Hence, the right-hand side is strictly positive as soon as $(-A, A) \cap$ $I_{0} \neq \emptyset$. The estimates presented in Propositions 4.1, 4.4, and 4.7 guarantee that the lefthand side tends to zero for $A \rightarrow \infty$. But the right-hand side is an increasing function of $A$ which takes positive values, so is bounded away from zero for large $A$. We thus reached a contradiction and conclude that the existence of a positive bounded solution of (48) implies that $\lambda_{\infty}>0$.

\subsection{Uniqueness of traveling waves}

Theorem 4.9. Equation (48) has at most one bounded positive solution.

Proof: The argument follows some ideas in Berestycki (1981). The new difficulty is that here we have to deal with an unbounded domain.

Assume there are two distinct solutions $w^{i}, i=1,2$. Writing the differential equation in the form

$$
\left(\mathrm{e}^{c x} w_{x}^{i}\right)_{x}+g\left(w^{i}(x), x\right) \mathrm{e}^{c x} w^{i}=0
$$

and manipulating as in the end of the proof of Theorem 4.8 we obtain for any $\alpha, \beta$ with $-\infty<\alpha<\beta<+\infty$, the identity

$$
\begin{aligned}
& {\left[-\mathrm{e}^{c x} w_{x}^{1} w^{2}+\mathrm{e}^{c x} w^{1} w_{x}^{2}\right]_{x=\alpha}^{x=\beta}} \\
& \quad=\int_{\alpha}^{\beta} \mathrm{e}^{c x}\left[g\left(w^{1}(x), x\right)-g\left(w^{2}(x), x\right)\right] w^{1}(x) w^{2}(x) d x .
\end{aligned}
$$

Now assume that $\left\{x: w^{2}(x)>w^{1}(x)\right\}$ is nonempty and let $(a, b)$ be a connected component of this set, then $w^{1}(a)=w^{2}(a)$ and $w^{1}(b)=w^{2}(b)$, where it is understood that $-\infty \leq a<b \leq+\infty$ and $w^{i}( \pm \infty)=0$ (recall Proposition 4.1). Suppose first that $a$ and $b$ are finite and take $\alpha=a$ and $\beta=b$. Then since $u \mapsto g(u, x)$ is decreasing, the right-hand side is positive. In fact, it is strictly positive (because the only way in which it could be zero is that $\left.g\left(w^{1}(x), x\right)=g\left(w^{2}(x), x\right)\right)$ for almost all $x \in(a, b)$, but then the $w^{i}$,s satisfy one and the same linear equation, as well as the same boundary conditions, so $w^{1} \equiv w^{2}$ on $(a, b))$. On the other hand, we must have that $w_{x}^{2}(a)>w_{x}^{1}(a)$ and $w_{x}^{2}(b)<w_{x}^{1}(b)$, so the left-hand side is strictly negative which is a contradiction. If either $\beta=+\infty$ or $\alpha=-\infty$ or both, we use the estimates of Propositions 4.1 and 4.4 to establish that the integral converges and that the corresponding terms at the left-hand side vanish in the limit $\beta \rightarrow \infty$ 
and/or $\alpha \rightarrow-\infty$. So, then too we arrive at the contradiction that the right-hand side is strictly positive while the left-hand side is at most zero.

Corollary 4.10. Equation (48) has exactly one bounded positive solution if $\lambda_{\infty}>0$ and no such solution if $\lambda_{\infty} \leq 0$.

\subsection{Large time behavior}

We now return to the evolution Eq. (1) and investigate the asymptotic behavior (for large time) of solutions of the initial value problem obtained by supplementing (1) by the initial condition

$$
u(0, x)=u_{0}(x)
$$

where $u_{0}$ is a given bounded nonnegative function defined on $\mathbb{R}$. Our assumptions on $g$ guarantee that the initial value problem has a unique, globally defined, solution $u=$ $u(t, x)$.

Theorem 4.11. Let $u$ be the solution of the Cauchy problem (1)-(59).

(i) If $\lambda_{\infty} \leq 0$, then $u(t, x) \rightarrow 0$ for $t \rightarrow \infty$, uniformly for $x \in \mathbb{R}$. That is, any population is bound to go extinct, no matter what the initial distribution is.

(ii) If $\lambda_{\infty}>0$ and $u_{0}$ is nontrivial, then $u(t, x)-w(x-c t) \rightarrow 0$ for $t \rightarrow \infty$, uniformly for $x \in \mathbb{R}$. Here, $w$ is the unique bounded positive solution of (48). So, any population is bound to persist by traveling along with the shifting climate.

Proof: Define $v(t, x):=u(t, x+c t)$ then (1) may be reformulated as

$$
v_{t}=v_{x x}+c v_{x}+f(v, x) .
$$

Let $M^{\prime}>\max \left\{M, \sup _{x} u_{0}(x)\right\}$ and let $z=z(t, x)$ be the solution of

$$
z_{t}=z_{x x}+c z_{x}+f(z, x), \quad z(0, x)=M^{\prime} .
$$

Since $M^{\prime}$ is a supersolution of the elliptic operator at the right-hand side of the differential equation, we know that $z_{t}<0$ (see, for instance Sattinger, 1973, p. 33). Since $z$ is bounded from below by zero, $z(t, \cdot)$ must converge for $t \rightarrow \infty$ to a nonnegative solution of (48). If $\lambda_{\infty} \leq 0$, the only such solution is zero. So, in that case, $z(t, x)$ converges to zero for $t \rightarrow \infty$. Additional arguments (explained in detail below) lead to the conclusion that the convergence is uniform for $x \in \mathbb{R}$. Since $0 \leq u(t, x)=v(t, x-c t) \leq z(t, x-c t)$, we conclude that if $\lambda_{\infty} \leq 0, u(t, x) \rightarrow 0$ for $t \rightarrow \infty$, uniformly for $x \in \mathbb{R}$.

It remains to prove (ii). If $u_{0}$ is nontrivial, $u(\delta, x)$ is strictly positive for $\delta>0$, and hence so is $v(\delta, x)$. So, for any given $R>0$ and $\varepsilon$ sufficiently small we have $v(\delta, x) \geq$ $\varepsilon \phi^{R}(x)$ for $-R \leq x \leq R$. Now assume that $\lambda_{\infty}>0$. Recall from the proof of Theorem 4.8 that for $R$ large enough, we obtain a subsolution if we extend $\varepsilon \phi^{R}(x)$ by zero outside the interval $[-R, R]$. Accordingly, $z(t, \cdot)$ cannot converge to zero for $t \rightarrow \infty$ when $\lambda_{\infty}>0$ and, therefore, the limit must be the unique bounded positive solution $w$ of (48), (compare 
Corollary 4.10). Likewise, the subsolution converges to $w$ and since $v$ is sandwiched in between; it too must converge to $w$.

We now show that the convergence is uniform for $x \in \mathbb{R}$. Again, we concentrate on the supersolution $z$. Suppose $z$ does not converge uniformly to $w$ for $t \rightarrow \infty$. This means that $\delta>0$ exists as well as sequences $t_{j} \rightarrow \infty$ and $x_{j} \in \mathbb{R}$ such that

$$
z\left(t_{j}, x_{j}\right)-w\left(x_{j}\right) \geq \delta .
$$

By possibly restricting to a subsequence, we may assume that

$$
x_{j} \rightarrow x_{\infty}, \quad \text { for } j \rightarrow \infty
$$

where $x_{\infty}$ is either finite, $+\infty$ or $-\infty$. Define

$$
z^{j}(t, x)=z\left(t, x+x_{j}\right)
$$

and note that for each $j, z^{j}$ is a decreasing function of $t$. Since $z^{j}$ is uniformly bounded, standard parabolic estimates guarantee that we can extract once more a subsequence, still denoted by $z^{j}$, such that $z^{j}$ converges uniformly on compact subsets to a function $z^{\infty}(t, x)$. Clearly $z^{\infty}$ is a nonincreasing function of $t$ and $z^{\infty}\left(t_{j}, 0\right)-w\left(x_{\infty}\right) \geq \delta$.

If $x_{\infty}$ is finite, then $z^{\infty}$ is a solution of

$$
z_{t}=z_{x x}+c z_{x}+f\left(z, x+x_{\infty}\right)
$$

and so its limit for $t \rightarrow \infty$ is a nontrivial solution of

$$
\tilde{w}_{x x}+c \tilde{w}_{x}+\tilde{w} g\left(\tilde{w}, x+x_{\infty}\right)=0 .
$$

By uniqueness, we must have

$$
\tilde{w}(x)=w\left(x+x_{\infty}\right)
$$

which, however, would imply that $\tilde{w}(0)=w\left(x_{\infty}\right)$ whereas taking the limit $j \rightarrow \infty$ in $z^{\infty}\left(t^{j}, 0\right)-w\left(x_{\infty}\right) \geq \delta$ we deduce that $\tilde{w}(0)-w\left(x_{\infty}\right) \geq \delta$. So, we ruled out the possibility that $x_{\infty}$ is finite.

Next, assume that $x_{\infty}=\infty$. Since $g(z, x) \leq g(0, x)$ and $g(0, x) \rightarrow-1$ for $x \rightarrow \infty$, we now deduce that $z^{\infty}$ satisfies the inequality

$$
z_{t} \leq z_{x x}+c z_{x}-z
$$

and once more taking the limit $t \rightarrow \infty$ that

$$
\tilde{w}_{x x}+c \tilde{w}_{x}-\tilde{w} \geq 0 .
$$

But a function satisfying this inequality cannot have a positive maximum, and hence no nontrivial bounded positive solution can exist. Since we must have $\tilde{w}(0) \geq \delta$ (note that $w\left(x_{j}\right) \rightarrow 0$ for $j \rightarrow \infty$ when $x_{\infty}=\infty$ ), we conclude that $x_{\infty}=\infty$ is impossible as well. The possibility that $x_{\infty}=-\infty$ is ruled out in exactly the same manner. 
So, the assumption that $z$ does not converge uniformly to $w$ for $t \rightarrow \infty$ leads to a contradiction and we conclude that the convergence is, in fact, uniform. The proof that the subsolution converges uniformly to $w$ follows exactly the same pattern. Hence, the true solution $v$, which lies in between, must converge uniformly to $w$ and the proof of (ii) is completed.

Finally, we note that the proof that $z(t, \cdot)$ converges uniformly to zero when $\lambda_{\infty} \leq 0$ is based on precisely the same arguments as used above.

\section{Concluding remarks}

Mathematical studies of simplified models can yield ecological insights, and at the same time, shed light on basic mechanisms. In that spirit, we have analyzed the effect that a shifting climate may have on the persistence of a species. A patch of favorable habitat, surrounded by unfavorable habitat, is able to sustain a population provided the gain by reproduction can balance the losses due to mortality inside the patch and dispersal away from the patch. If the patch itself moves in space, an additional loss term is created, since individuals may be left behind. Dispersing individuals, on the other hand, may be fortunate enough to land where conditions are changing for the better. As a result, the critical size that a patch should have in order to sustain a population, does not only depend on reproduction, mortality and dispersal rates, but also on the speed with which the patch moves through space. In Section 2, we have derived an explicit expression in formulas (23), (24), and (25) for the dependence which produces valuable insights. In Section 4 we have rigorously established several mathematical properties for a large class of models.

Persistence in a moving patch is facilitated when the rate of climate change is low, the rate of population growth within the patch is high, and the climate outside the patch not too hostile (Fig. 5). Migration, however, is a double-edged sword. Both too much and too little dispersal can lead to extinction and the optimal dispersal rate increases with patch speed (Fig. 5). The results imply that a small latitudinal range diminishes the maximal rate of climate change a species should be able to track. This means that the conventional approach (see Skellam, 1951) of using the invasion (Fisher) speed as an estimate of this maximal rate can lead to a severe overestimation when ranges are small or $D$ is large.

A moving climate can have dramatic effects on the size and form of the population profile. When the favorable region moves to the north, the population becomes more concentrated toward the north end of the population profile. Interestingly, if the habitat outside the favorable patch is not too hostile, the south tail becomes considerably thicker and longer as a result of the movement, since it takes a while before the marooned local population disappears. As a consequence, movement may result in increases in both the total population size and the population range (Figs. 7, 8 and 9).

In unpublished simulations of a metapopulation model, Nagelkerke (2004) obtained results similar to those reported here on our continuous population model. This demonstrates the structural robustness of our sometimes counterintuitive findings. For example, he modeled jump dispersal of propagules. This leads us to believe that our results are not restricted to movement by simple diffusion. Distance dispersal is relevant for many organisms. 
Here, we have concentrated on the long time dynamics. Nagelkerke (2004) also studied the transient dynamics shortly after the climate starts to move. He found that generally the northern border initially moves faster than the southern border, both for surviving populations and for those that were doomed to go extinct. In the case of ultimate extinction, the southern border catches up after a while and then moves even faster than the climate, until it collides with the northern border. Note that another reason for not being too confident about an increasing range is the threshold behavior shown in Fig. 8. A small additional increase in climate speed can cause total collapse. The initial asymmetry between the velocities of both borders is in agreement with the outcome of an extensive analysis of butterfly data by Parmesan et al. (1999) that found more evidence for moving northern borders than for southern borders, suggesting that this is a transient phenomenon (see also Collingham et al., 1996). In addition, it could be easier to observe the move of the steep north front than that of the far less steep south back.

Our analysis was relatively simple, since we considered a one-dimensional spatial domain. Two-dimensional models give rise to new subtleties. Some mathematical issues involved in higher-dimensional versions of this problem will be discussed in Berestycki and Rossi (2008). Of particular interest is to understand the effect of the geometry on the ability to persist despite a climate change. For instance, a bottle-neck may occur when the extension of the patch in the lateral direction has a local minimum-giving rise to a narrow strait. Actually, one could mimic this effect in the one-dimensional setting by allowing the diffusion coefficient to depend on the spatial variable $\mathrm{x}$; there would then be both an $\mathrm{x}$ and an $\mathrm{x}$-ct dependence, making the problem inhomogeneous even modulo time translation. We plan to analyze such problems in further works.

\section{Acknowledgements}

Part of the research presented in this paper was carried out while Henri Berestycki was visiting the Department of Mathematics at the University of Chicago, which he thanks for its hospitality. Odo Diekmann thanks Frithjof Lutscher for bringing the work of Potapov and Lewis to his attention and A.B. Potapov for stimulating discussions during a Spatial Ecology meeting in Miami organized by Cantrell, Cosner, and DeAngelis. Lastly, the authors are indebted to Lionel Roques (INRA, Avignon, France), for providing the computations shown in Figs. 5, 6, 7, and 8.

Open Access This article is distributed under the terms of the Creative Commons Attribution Noncommercial License which permits any noncommercial use, distribution, and reproduction in any medium, provided the original author(s) and source are credited.

\section{References}

Aronson, D.G., Weinberger, H.F., 1978. Multidimensional nonlinear diffusions arising in population genetics. Adv. Math. 30, 33-76.

Berestycki, H., 1981. Le nombre de solutions de certains problèmes semi-linéaires elliptiques. J. Funct. Anal. 40, 1-29.

Berestycki, H., Hamel, F., 2009. Reaction-Diffusion Equations and Propagation Phenomena. Springer, New York, to appear. 
Berestycki, H., Nirenberg, L., 1991. Asymptotic behavior via the Harnack inequality. In: Ambrosetti, A. (Ed.), Nonlinear Analysis, A tribute in honor of G. Prodi, Quaderni Sc. Norm. Sup. Pisa, pp. 135-144.

Berestycki, H., Rossi, L., 2008. Reaction-diffusion equations for population dynamics with forced speed, I-The case of the whole space. Discrete Contin. Dyn. Syst. A 21, 41-67.

Berestycki, H., Nirenberg, L., Varadhan, S.R.S., 1994. The principal eigenvalue and maximum principle for second order elliptic operators in general domains. Commun. Pure Appl. Math. 47, 47-92.

Berestycki, H., Hamel, F., Roques, L., 2005a. Analysis of the periodically fragmented environment model: I-Species persistence. J. Math. Biol. 51, 75-113.

Berestycki, H., Hamel, F., Roques, L., 2005b. Analysis of the periodically fragmented environment model: II-Biological invasions and pulsating traveling fronts. J. Math. Pures Appl. 84, 1101-1146.

Berestycki, H., Hamel, F., Rossi, L., 2007. Liouville-type results for semilinear elliptic equations in unbounded domains. Ann. Mat. Pura Appl. 186, 469-507.

Cano-Casanova, S., López-Gómez, J., 2003. Permanence under strong aggressions is possible. Ann. Inst. H. Poincaré, Anal. Non Linéaire 20, 999-1041.

Cantrell, R.S., Cosner, C., 1991. The effects of spatial heterogeneity in population dynamics. J. Math. Biol. 29, 315-338.

Cantrell, R.S., Cosner, C., 1998. On the effects of spatial heterogeneity on the persistence of interacting species. J. Math. Biol. 37, 103-145.

Cantrell, R.S., Cosner, C., 2003. Spatial Ecology Via Reaction-Diffusion Equations. Wiley, New York.

Collingham, Y.C., Hill, M.O., Huntley, B., 1996. The migration of sessile organisms: a simulation model with measurable parameters. J. Veg. Sci. 7, 831-846.

Dahmen, K.A., Nelson, D.R., Shnerb, N.M., 2000. Life and death near a windy oasis. J. Math. Biol. 41, $1-23$.

Deasi, M.N., Nelson, D.R., 2005. A quasispecies on a moving oasis. Theor. Pop. Biol. 67, 33-45.

Diekmann, O., Temme, N.M. (Eds.) 1982. Nonlinear diffusion problems. MC Syllabus, 28, Amsterdam.

Fisher, R.A., 1937. The advance of advantageous genes. Ann. Eugen. 7, 335-369.

Gilbarg, D., Trudinger, N.S., 1998. Elliptic Partial Differential Equations of Second Order. Springer, New York.

Hadeler, K.P., Rothe, F., 1975. Travelling fronts in nonlinear diffusion equations. J. Math. Biol. 2, 251263.

Kolmogorov, A.N., Petrovsky, I.G., Piskunov, N.S., 1937. Étude de l'équation de la diffusion avec croissance de la quantité de matière et son application à un problème biologique, Bulletin Université d'État à Moscou (Bjul. Moskowskogo Gos. Univ.), Série internationale A 1, pp. 1-26.

Krylov, N.V., 1987. Nonlinear Elliptic and Parabolic Equations of the Second Order. Reidel, Dordrecht.

Ladyženskaja, O.A., Solonnikov, V.A., Ural'ceva, N.N., 1968. Linear and Quasilinear Equations of Parabolic Type. AMS, Providence.

Ludwig, D., Aronson, D.G., Weinberger, H.F., 1979. Spatial patterning of the spruce budworm. J. Math. Biol. 8, 217-258.

Murray, J.D., Sperb, R.P., 1983. Minimum domains for spatial patterns in a class of reaction-diffusion equations. J. Math. Biol. 18, 169-184.

Nagelkerke, C.J., 2004. Unpublished.

Okubo, A., Levin, S.A., 2001. Diffusion and Ecological Problems: Modern Perspectives, 2nd edn. Springer, Berlin.

Pachepsky, E., Lutscher, F., Nisbet, R.M., Lewis, M.A., 2005. Persistence, spread and the drift paradox. Theor. Popul. Biol. 67, 61-73.

Parmesan, C., Ryrholm, N., Srtefanescu, C., Hill, J.K., Thomas, C.D., Descimon, H., Huntley, B., Kaila, L., Kullberg, J., Tammaru, T., Tennent, W.J., Thomas, J.A., Warren, M., 1999. Poleward shifts in geographical ranges of butterfly species associated with regional warming. Nature 399, 579-583.

Pease, C.M., Lande, R., Bull, J.J., 1989. A model of population growth, dispersal and evolution in a changing environment. Ecology 70, 1657-1664.

Pinsky, R.G., 1995. Positive Harmonic Functions and Diffusion. Cambridge University Press, Cambridge.

Potapov, A.B., Lewis, M.A., 2004. Climate and competition: the effect of moving range boundaries on habitat invasibility. Bull. Math. Biol. 66, 975-1008.

Rass, L., Radcliffe, J., 2003. Spatial Deterministic Epidemics. Mathematical Surveys and Monographs, vol. 102. Am. Math. Sos., Providence.

Roques, L., Stoica, R.S., 2007. Species persistence decreases with habitat fragmentation: an analysis in periodic stochastic environments. J. Math. Biol. 55, 189-205.

Sattinger, D.H., 1973. Topics in Stability and Bifurcation Theory. Springer, Berlin. 
Schaaf, R., 1990. Global Solution Branches of Two Point Boundary Value Problems. Springer LNM, vol. 1458.

Shi, J., Shivaji, R., 2006. Persistence in diffusion models with weak Allee effect. J. Math. Biol. 52, 807829.

Shigesada, N., Kawasaki, K., 1997. Biological Invasions: Theory and Practice. Oxford Series in Ecology and Evolution. Oxford University Press, Oxford.

Shigesada, N., Kawasaki, K., Teramoto, E., 1986. Traveling periodic waves in heterogeneous environments. Theor. Popul. Biol. 30, 143-160.

Skellam, J.G., 1951. Random dispersal in theoretical populations. Biometrika 38, 196-218.

Thieme, H.R., Zhao, X.-Q., 2003. Asymptotic speeds of spread and traveling waves for integral equations and delayed reaction-diffusion models. J.D.E. 195, 430-470.

Volpert, A.I., Volpert, V.A., Volpert, V.A., 1994. Traveling Wave Solutions of Parabolic Systems. Translations of Math. Monographs, vol. 140. Am. Math. Soc., Providence.

Walther, G.-R., Post, E., Convey, P., Menzel, A., Parmesan, C., Beebee, T.J.C., Fromentin, J.-M., HoeghGuldberg, O., Bairlein, F., 2002. Ecological responses to recent climate change. Nature 416, 389-395.

Weinberger, H., 2002. On spreading speed and traveling waves for growth and migration models in a periodic habitat. J. Math. Biol. 45, 511-548. 